\title{
Testing the AdS/CFT correspondence by Monte Carlo calculation of BPS and non-BPS Wilson loops in $\mathbf{4 d} \mathscr{N}=4$ super Yang-Mills theory
}

\author{
Masazumi Honda $^{* a}$, Goro Ishiki ${ }^{b}$, Jun Nishimura ${ }^{a, c}$ and Asato Tsuchiya ${ }^{d}$ \\ ${ }^{a}$ Department of Particle and Nuclear Physics, \\ Graduate University for Advanced Studies (SOKENDAI), \\ Tsukuba, Ibaraki 305-0801, Japan \\ ${ }^{b}$ Department of Physics, Kyoto University, \\ 3 Kyoto 606-8502, Japan \\ ${ }^{c}$ KEK Theory Center, High Energy Accelerator Research Organization (KEK), \\ Tsukuba, Ibaraki 305-0801, Japan \\ ${ }^{d}$ Department of Physics, Shizuoka University, \\ 836 Ohya, Suruga-ku, Shizuoka 422-8529, Japan \\ mhondaepost.kek.jplishiki@post.kek.jp. \\ jnishi@post.kek.jp,satsuch@ipc.shizuoka.ac.jp
}

We test the AdS/CFT correspondence by calculating Wilson loops in $\mathscr{N}=4$ super Yang-Mills theory on $R \times S^{3}$ in the planar limit. Our method is based on a novel large- $N$ reduction, which reduces the problem to Monte Carlo calculations in the plane-wave matrix model or the BMN matrix model, which is a $1 \mathrm{~d}$ gauge theory with 16 supercharges. By using the gauge-fixed momentumspace simulation, we obtain results respecting 16 supersymmetries. We report on the Monte Carlo results for the BPS circular Wilson loop, which reproduce the exact result up to strong coupling. As a future prospect, we calculate a track-shaped Wilson loop from the gravity side, which shows that a clear test of the AdS/CFT for the non-BPS case is also feasible.

The XXIX International Symposium on Lattice Field Theory, Lattice2011

July 10-16, 2011

Squaw Valley Lake Tahoe, California

\footnotetext{
${ }^{*}$ Speaker.
} 


\section{Introduction}

The gauge-gravity duality [1] has been one of the most important subjects in string theory over the past decade. The most typical example is the so-called AdS/CFT correspondence between type IIB superstring theory on $A d S_{5} \times S^{5}$ and $4 \mathrm{~d} \mathscr{N}=4 \mathrm{SU}(N)$ super Yang-Mills theory (SYM). Even in this case, however, a complete proof of the duality is still missing. One of the reasons is that the parameter region described by the classical supergravity on the string theory side corresponds to the strongly coupled region in the planar large- $N$ limit on the gauge theory side. In order to study the strongly coupled $4 \mathrm{~d} \mathscr{N}=4 \mathrm{SYM}$ from first principles, one needs to have a non-perturbative formulation such as the lattice QCD. The problem here is that the lattice regularization necessarily breaks translational symmetry, which is included in the supersymmetry (SUSY). In order to restore SUSY in the continuum limit, one generally has to fine-tune parameters in the lattice action ${ }^{1}$.

In this work we adopt an alternative regularization method based on the idea of the large$N$ reduction [3], which preserves 16 supersymmetries. Since $4 \mathrm{~d} \mathscr{N}=4 \mathrm{SYM}$ has conformal symmetry, the theory on $R^{4}$ is equivalent to the theory on $R \times S^{3}$ through conformal mapping. The novel large- $N$ reduction [4] relates this theory to a reduced model, which can be obtained by shrinking the $S^{3}$ to a point. The resulting one-dimensional gauge theory with 16 supercharges can be studied by using the gauge-fixed momentum-space simulation [5] as in recent studies of the D0brane system [6]. Thus we can perform Monte Carlo calculations in 4d $\mathscr{N}=4$ SYM respecting SUSY maximally and without fine-tuning ${ }^{2}$.

We are going to test the AdS/CFT correspondence by calculating BPS and non-BPS Wilson loops in $4 \mathrm{~d} \mathscr{N}=4 \mathrm{SYM}^{3}$. In particular, we reproduce an exact result for the circular Wilson loop, which serves as a check of our method. For a non-trivial test of the AdS/CFT correspondence, we consider the track-shaped Wilson loop as an example of non-BPS operators, which cannot be calculated by analytic methods relying on SUSY. We calculate it on the gravity side by numerically solving a classical string equation of motion.

\section{Large- $N$ reduction for $\mathscr{N}=4 \mathrm{SYM}$ on $R \times S^{3}$}

Let us first discuss the novel large- $N$ reduction for $\mathscr{N}=4 \mathrm{SYM}$ on $R \times S^{3}$. By collapsing the $S^{3}$ to a point, we obtain the plane wave matrix model (PWMM) or the BMN matrix model [10 $]^{4}$, whose action is given by

$$
\begin{gathered}
S_{\mathrm{PW}}=\frac{1}{g_{\mathrm{PW}}^{2}} \int d t \operatorname{tr}\left[\frac{1}{2}\left(D_{t} X_{M}\right)^{2}-\frac{1}{4}\left[X_{M}, X_{N}\right]^{2}+\frac{1}{2} \Psi^{\dagger} D_{t} \Psi-\frac{1}{2} \Psi^{\dagger} \gamma_{M}\left[X_{M}, \Psi\right]\right. \\
\left.+\frac{\mu^{2}}{2}\left(X_{i}\right)^{2}+\frac{\mu^{2}}{8}\left(X_{a}\right)^{2}+i \mu \varepsilon_{i j k} X_{i} X_{j} X_{k}+i \frac{3 \mu}{8} \Psi^{\dagger} \gamma_{123} \Psi\right]
\end{gathered}
$$

Here the parameter $\mu$ is related to the radius of $S^{3}$ as $R_{S^{3}}=\frac{2}{\mu}$, and the covariant derivative is defined by $D_{t}=\partial_{t}-i[A, \cdot]$, where $A(t)$, as well as $X_{M}(t)$ and $\Psi(t)$, is an $N \times N$ hermitian matrix. The

\footnotetext{
${ }^{1}$ Recently it was claimed that fine-tuning can be avoided at least up to the 1-loop level by using a lattice formulation with topological twist [2].

${ }^{2}$ See refs. [7] for proposals for finite $N$.

${ }^{3}$ See refs. [8, 9] for some preliminary results on the Wilson loop and correlation functions.

${ }^{4}$ Properties of this model at finite temperature are studied at weak coupling [11, 12] and at strong coupling [13].
} 
range of indices is given by $1 \leq M, N \leq 9,1 \leq i, j, k \leq 3$ and $4 \leq a \leq 9$. The model has the $\mathrm{SU}(2 \mid 4)$ symmetry with 16 supercharges.

The PWMM possesses many discrete vacua representing multi fuzzy spheres, which are given explicitly by

$$
X_{i}=\mu \bigoplus_{I=1}^{v}\left(L_{i}^{\left(n_{I}\right)} \otimes \mathbf{1}_{k_{I}}\right) \quad \text { with } \sum_{I=1}^{v} n_{I} k_{I}=N
$$

where $L_{i}^{(r)}$ are the $r$-dimensional irreducible representation of the $\mathrm{SU}(2)$ algebra $\left[L_{i}^{(r)}, L_{j}^{(r)}\right]=$ $i \varepsilon_{i j k} L_{k}^{(r)}$. These vacua preserve the $\mathrm{SU}(2 \mid 4)$ symmetry, and are all degenerate.

In order to retrieve the planar $\mathscr{N}=4 \mathrm{SYM}$ on $R \times S^{3}$, one has to pick up a particular background from (2.2), and consider the theory (2.1) around it. Let us consider the vacuum defined by

$$
k_{I}=k, \quad n_{I}=n+I-\frac{v+1}{2} \quad \text { for } I=1, \cdots, v,
$$

and take the large- $N$ limit in such a way that

$$
k \rightarrow \infty, \frac{n}{v} \rightarrow \infty, v \rightarrow \infty, \quad \text { with } \lambda_{\mathrm{PW}} \equiv \frac{g_{\mathrm{PW}}^{2} k}{n} \text { fixed } .
$$

Then the resulting theory is claimed [4] to be equivalent ${ }^{5}$ to the planar limit of $\mathscr{N}=4 \mathrm{SYM}$ on $R \times S^{3}$ with the 't Hooft coupling constant given by

$$
\lambda_{\mathrm{SYM}}=2 \pi^{2} \lambda_{\mathrm{PW}}\left(R_{S^{3}}\right)^{3}=\frac{16 \pi^{2} k}{n} \frac{g_{\mathrm{PW}}^{2}}{\mu^{3}} .
$$

The above equivalence may be viewed as an extension of the large- $N$ reduction [3], which asserts that the large- $N$ gauge theories can be studied by dimensionally reduced models. The original idea for theories compactified on a torus can fail due to the instability of the $\mathrm{U}(1)^{D}$ symmetric vacuum of the reduced model [16]. This problem is avoided in the novel proposal since the PWMM is a massive theory and the vacuum preserves the maximal SUSY. This regularization respects 16 supersymmetries, which is half of the full superconformal symmetry of $\mathscr{N}=4 \mathrm{SYM}$ on $R \times S^{3}$. Since any kind of UV regularization breaks the conformal symmetry, this regularization is optimal from the viewpoint of preserving SUSY.

\section{Wilson loops in 4d $\mathscr{N}=4 \mathrm{SYM}$}

Let us consider the following type of Wilson loop

$$
W(C)=\frac{1}{N} \operatorname{Tr} \mathscr{P} \exp \oint_{C} d s\left(i A_{\mu}^{R^{4}} \dot{x}^{\mu}(s)+\left|\dot{x}^{\mu}(s)\right| X_{a}^{R^{4}} \theta_{a}\right),
$$

where $\dot{x}^{\mu}(s) \equiv \frac{d x^{\mu}(s)}{d s}$ and $\theta_{a}$ is a constant which satisfies $\theta_{a} \theta_{a}=1$. The fields $A_{\mu}^{R^{4}}$ and $X_{a}^{R^{4}}$ represent the gauge field and the six scalars in $4 \mathrm{~d} \mathscr{N}=4 \mathrm{SYM}$ on $R^{4}$, respectively. Due to the particular way

\footnotetext{
${ }^{5}$ See refs. [14] for earlier studies that led to this proposal. This equivalence was checked at finite temperature in the weak coupling regime [12]. It has also been extended to general group manifolds and coset spaces [15].
} 
in which the scalars appear, one can obtain predictions from the gravity side based on the AdS/CFT correspondence as

$$
\lim _{N \rightarrow \infty, \lambda_{\mathrm{SYM}} \rightarrow \infty}\langle W(C)\rangle_{\mathrm{SYM}}=e^{-S(C)},
$$

where $S(C)$ represents the area of the minimal surface spanning the loop $C$ on the boundary of the AdS space.

For the circular Wilson loop $W\left(C_{\text {circ }}\right)$, which is a $(1 / 2-)$ BPS operator, there is an exact result on the gauge theory side, which is obtained by summing up planar ladder diagrams or by using the localization method [17]. The result is given by

$$
\begin{aligned}
\lim _{N \rightarrow \infty}\left\langle W\left(C_{\text {circ }}\right)\right\rangle_{\mathrm{SYM}} & =\sqrt{\frac{2}{\lambda_{\mathrm{SYM}}}} I_{1}\left(\sqrt{2 \lambda_{\mathrm{SYM}}}\right) \\
& \simeq \frac{e^{\sqrt{2 \lambda_{\mathrm{SYM}}}}}{\left(\frac{\pi}{2}\right)^{1 / 2}\left(2 \lambda_{\mathrm{SYM}}\right)^{3 / 4}} \quad \text { for } \lambda_{\mathrm{SYM}} \gg 1,
\end{aligned}
$$

where $I_{1}(x)$ is the modified Bessel function of the first kind. The result is independent on the radius of the circle, which is a consequence of the scale invariance of $\mathscr{N}=4$ SYM. At strong coupling it agrees with the result obtained from the dual geometry $S\left(C_{\text {circ }}\right)=-\sqrt{2 \lambda_{\mathrm{SYM}}}[18]$. This is an explicit example of the AdS/CFT correspondence. We use the exact result (3.3) for arbitrary $\lambda_{\text {SYM }}$ to check our calculation method.

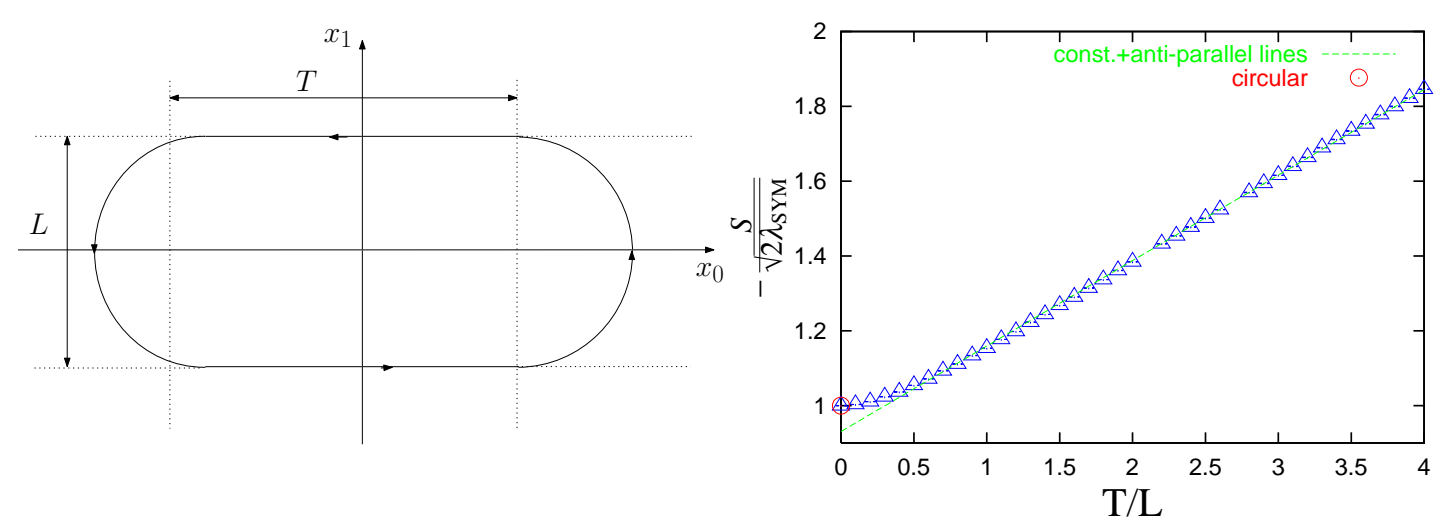

Figure 1: (Left) The track-shaped Wilson loop has two straight lines of length $T$, which are anti-parallel to each other, and two semi-circles with radius $L / 2$. (Right) The numerical results for $-\frac{S\left(C_{\text {track }(T, L)}\right)}{\sqrt{2 \lambda_{\mathrm{SYM}}}}$ is plotted as a function of $T / L$. The circle at $T=0$ represents the circular limit $(T \rightarrow 0)$ and the straight line represents a fit to the anti-parallel-line limit $(T \rightarrow \infty)$ with an additive constant treated as a free parameter.

The track-shaped Wilson loop has two parameters $T$ and $L$ as depicted in figure 1 (Left). It has two limits: the $T \rightarrow 0$ limit, which corresponds to the circular Wilson loop, and $T \rightarrow \infty$, which corresponds to two anti-parallel lines. Since the track-shaped Wilson loop for $T \neq 0$ is a nonBPS operator, it cannot be calculated on the gauge theory side by such analytic methods as the localization method which rely on SUSY. The limit $(T \rightarrow \infty)$ of two anti-parallel lines is calculated based on the AdS/CFT correspondence from the gravity side as [19]

$$
\lim _{T \rightarrow \infty} \frac{1}{T} \ln \left\langle W\left(C_{\operatorname{track}(T, L)}\right)\right\rangle_{\mathrm{SYM}}=-\sqrt{2 \lambda_{\mathrm{SYM}}} \frac{4 \pi^{2}}{\Gamma(1 / 4)^{4}} \frac{1}{L},
$$


which is consistent with the conformal symmetry of the theory, and also with the fact that $\mathscr{N}=4$ SYM on $R^{4}$ is in the Coulomb phase. Here we extend the calculation on the gravity side to arbitrary $T / L$, and obtain explicit numerical results by solving the classical string equation of motion. In figure 1 (Right) we plot the minimal surface obtained by Newton's method.

\section{Monte Carlo method}

In order to simulate the PWMM (2.1), we compactify the $t$-direction to a circle of circumference $\beta$. Since we are interested in the properties at zero temperature, we impose periodic boundary conditions on both scalars $X_{M}(t)$ and fermions $\Psi_{\alpha}(t)$, which keep SUSY intact. In Fourier-mode simulation [5], we first fix the gauge symmetry completely by choosing $A(t)=\frac{1}{\beta} \operatorname{diag}\left(\alpha_{1}, \cdots, \alpha_{N}\right)$ with $-\pi<\alpha_{a} \leq \pi$, and then make a Fourier expansion $X_{M}(t)=\sum_{n=-\Lambda}^{\Lambda} \tilde{X}_{M, n} e^{i \omega n t}\left(\omega \equiv \frac{2 \pi}{\beta}\right)$ and similarly for the fermions. The upper bound $\Lambda$ on the Fourier modes plays the role of the UV cutoff. The original PWMM can be retrieved by just taking the limits $\beta \rightarrow \infty$ and $\frac{\Lambda}{\beta} \rightarrow \infty$ since there are neither UV nor IR divergences. The model regularized by finite $\beta$ and $\Lambda$ can be simulated by the RHMC algorithm. This method has been applied extensively to the D0-brane system corresponding to $\mu=0$, and the results confirmed the gauge/gravity duality for various observables [6]. ${ }^{6}$ Since the parameter $\mu$ in the action (2.1) can be scaled away by appropriate redefinition of fields and parameters, we take $\mu=2\left(R_{S^{3}}=1\right)$ without loss of generality.

The Wilson loop in $\mathscr{N}=4 \mathrm{SYM}$ can be calculated in PWMM in the following way. When we perform the conformal mapping from $R^{4}$ to $R \times S^{3}$, the radial and angular directions are mapped to the time and $S^{3}$-directions, respectively. Therefore, an arbitrary loop on a plane in $R^{4}$ is mapped to a loop on $R \times S^{3}$, which can be projected to a great circle on $S^{3}$. Such a Wilson loop can be represented in the large- $N$ reduced model as

$$
W_{\mathrm{red}}(C)=\frac{1}{N} \operatorname{Tr} \mathscr{P} \exp \oint_{C} d s\left(i A_{0} \frac{d t}{d s}+i X_{i} e_{\mu}^{i} \dot{x}^{\mu}(s)+\left|\dot{x}_{\mu}(s)\right| X_{a} \theta_{a}\right),
$$

where $e_{j}^{i}=e_{j}^{i}\left(x^{\mu}(s)\right)$ is the dreibein on $S^{3}$. The expectation value of this operator is related to the average of the original Wilson loop as [21]

$$
\langle W(C)\rangle_{\mathrm{SYM}}=\left\langle W_{\text {red }}(C)\right\rangle,
$$

where $\langle\cdots\rangle$ on the right-hand side denotes the expectation value in the large- $N$ reduced model (PWMM). In the case of circular Wilson loop, the relation (4.2) was confirmed by reproducing the SYM result (3.3) from the reduced model to all orders in perturbation theory assuming that non-ladder diagrams do not contribute [22].

\section{Numerical results}

In figure 2 we present our preliminary results for the circular Wilson loop. We have performed the $\Lambda \rightarrow \infty$ extrapolation using $\Lambda=6,8,10,12$ assuming that finite $\Lambda$ effects are $\mathrm{O}(1 / \Lambda)$. The background is chosen to be $(n, v)=\left(\frac{3}{2}, 2\right)$ and we performed an extrapolation to $k=\infty$ using the

\footnotetext{
${ }^{6}$ See refs. [20] for Monte Carlo calculations based on the lattice regularization.
} 
data for $k=2,3,4,5$ assuming that the finite-k effects are $\mathrm{O}\left(1 / k^{2}\right)$. We also plot the exact result (3.3). Except for the data point at $\sqrt{\lambda_{\mathrm{SYM}}}=4$, the agreement with the exact result is promising. Note, in particular, that we already start to observe a bent from the weak coupling behavior towards the strong coupling behavior. This is remarkable considering the rather small matrix size. We consider this as a result of the fact that our formulation respects sixteen supersymmetries.

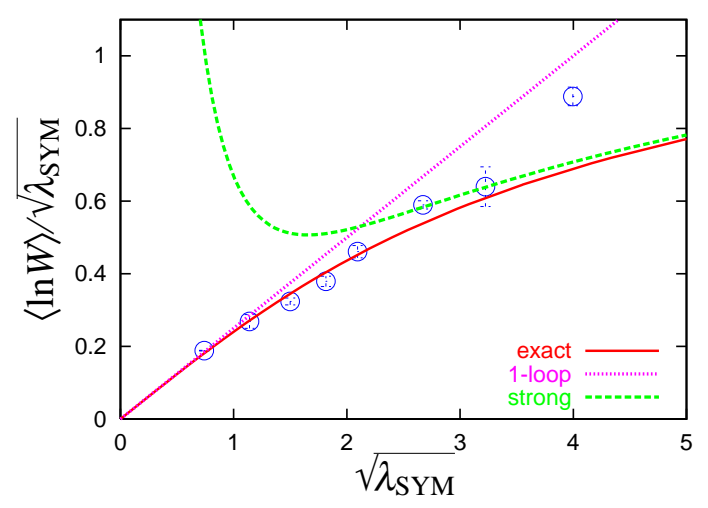

\begin{tabular}{|c||c|c|c|c|c|c|c|c|}
\hline$g_{\mathrm{PW}}^{2} N$ & 0.13 & 0.30 & 0.51 & 0.75 & 1.00 & 1.63 & 2.37 & 3.64 \\
\hline$\beta$ & 10.0 & 7.50 & 6.25 & 5.50 & 5.00 & 4.25 & 3.75 & 3.25 \\
\hline \hline$\lambda_{\text {SYM }}$ & 0.55 & 1.30 & 2.25 & 3.30 & 4.39 & 7.14 & 10.4 & 16.0 \\
\hline
\end{tabular}

Figure 2: The log of the circular Wilson loop normalized by $\sqrt{\lambda_{\mathrm{SYM}}}$ is plotted against $\sqrt{\lambda_{\mathrm{SYM}}}$. The values of $g_{\mathrm{PW}}^{2} N$ and $\beta$ (and the corresponding values of $\lambda_{\mathrm{SYM}}$ ) we use are listed in the table. The solid line represents the exact result (3.3). The dashed line represents the behavior (3.4) at strong coupling, whereas the dotted line represents the leading perturbative behavior $\ln \langle W\rangle \simeq \frac{1}{4} \lambda_{\text {SYM }}$.

\section{Summary and discussions}

We have investigated nonperturbative properties of the $4 \mathrm{~d} \mathrm{SU}(\infty) \mathscr{N}=4 \mathrm{SYM}$ from firstprinciples respecting 16 supersymmetries. In particular, we have reproduced the exact result (3.3) up to $\lambda_{\mathrm{SYM}} \simeq 10.4$. As a nontrivial check of the AdS/CFT correspondence, we are planning to study the track-shaped Wilson loop. We have obtained explicit results on the gravity side by numerically solving a classical string equation of motion. The results nicely interpolate the two limits, which are already calculated, i.e., the circular loop and the anti-parallel lines. We hope to report on the calculations on the gauge theory side in the forth-coming publication.

\section{Acknowledgments}

We thank H. Kawai and Y. Kitazawa for valuable discussions. Computation was carried out on PC clusters at KEK and Fermilab. The work of M. H. is supported by Japan Society for the Promotion of Science (JSPS). The work of G. I. is supported by the Grant-in-Aid for the Global COE Program "The Next Generation of Physics, Spun from Universality and Emergence" from the 
Ministry of Education, Culture, Sports, Science and Technology (MEXT) of Japan. The work of J. N. and A. T. is supported by Grant-in-Aid for Scientific Research (No. 19340066, 20540286, 19540294 and 23244057) from JSPS.

\section{References}

[1] J. M. Maldacena, Adv. Theor. Math. Phys. 2 (1998) 231.

[2] S. Catterall, E. Dzienkowski, J. Giedt, A. Joseph, R. Wells, JHEP 1104, 074 (2011).

[3] T. Eguchi and H. Kawai, Phys. Rev. Lett. 48 (1982) 1063.

[4] T. Ishii, G. Ishiki, S. Shimasaki and A. Tsuchiya, Phys. Rev. D 78 (2008) 106001.

[5] M. Hanada, J. Nishimura and S. Takeuchi, Phys. Rev. Lett. 99 (2007) 161602.

[6] K. N. Anagnostopoulos, M. Hanada, J. Nishimura and S. Takeuchi, Phys. Rev. Lett. 100 (2008) 021601; M. Hanada, A. Miwa, J. Nishimura and S. Takeuchi, Phys. Rev. Lett. 102 (2009) 181602; M. Hanada, Y. Hyakutake, J. Nishimura and S. Takeuchi, Phys. Rev. Lett. 102 (2009) 191602; M. Hanada, J. Nishimura, Y. Sekino and T. Yoneya, Phys. Rev. Lett. 104 (2010) 151601.

[7] M. Hanada, S. Matsuura and F. Sugino, arXiv:1004.5513 [hep-lat]; M. Hanada, arXiv:1009.0901 [hep-lat].

[8] J. Nishimura, PoS LAT2009 (2009) 016.

[9] M. Honda, G. Ishiki, S. W. Kim, J. Nishimura and A. Tsuchiya, PoSLATTICE 2010, 253 (2010) .

[10] D. E. Berenstein, J. M. Maldacena and H. S. Nastase, JHEP 0204 (2002) 013.

[11] N. Kawahara, J. Nishimura and K. Yoshida, JHEP 0606 (2006) 052.

[12] G. Ishiki, S. W. Kim, J. Nishimura and A. Tsuchiya, Phys. Rev. Lett. 102 (2009) 111601; JHEP 0909 (2009) 029; Y. Kitazawa and K. Matsumoto, Phys. Rev. D 79 (2009) 065003.

[13] S. Catterall and G. van Anders, JHEP 1009 (2010) 088.

[14] G. Ishiki, S. Shimasaki, Y. Takayama and A. Tsuchiya, JHEP 0611 (2006) 089; T. Ishii, G. Ishiki, S. Shimasaki and A. Tsuchiya, JHEP 0705 (2007) 014; Phys. Rev. D 77 (2008) 126015.

[15] H. Kawai, S. Shimasaki and A. Tsuchiya, Int. J. Mod. Phys. A 25 (2010) 3389; Phys. Rev. D 81 (2010) 085019.

[16] G. Bhanot, U. M. Heller and H. Neuberger, Phys. Lett. B 113 (1982) 47.

[17] J. K. Erickson, G. W. Semenoff, K. Zarembo, Nucl. Phys. B582, 155-175 (2000), N. Drukker, D. J. Gross, J. Math. Phys. 42, 2896-2914 (2001), V. Pestun, [arXiv:0712.2824 [hep-th]].

[18] D. E. Berenstein, R. Corrado, W. Fischler and J. M. Maldacena, Phys. Rev. D 59, 105023 (1999)

[19] J. M. Maldacena, Phys. Rev. Lett. 80, 4859-4862 (1998), S. -J. Rey, J. -T. Yee, Eur. Phys. J. C22, 379-394 (2001).

[20] S. Catterall and T. Wiseman, JHEP 0712 (2007) 104; Phys. Rev. D 78 (2008) 041502; JHEP 1004 (2010) 077.

[21] T. Ishii, G. Ishiki, K. Ohta, S. Shimasaki, A. Tsuchiya, Prog. Theor. Phys. 119, 863-882 (2008).

[22] G. Ishiki, S. Shimasaki, A. Tsuchiya, JHEP 1111, 036 (2011). 